\title{
THE SOLUTION OF A PAIR OF DUAL INTEGRAL EQUATIONS $\dagger$
}

\author{
by M. LOWENGRUB and I. N. SNEDDON \\ (Received 8 December, 1961)
}

1. We define two functions $F(\rho), G(\rho)$ in terms of a third function $\psi(\xi)$ by the equations

$$
F(\rho)=\int_{0}^{\infty} \xi^{n} \psi(\xi) J_{v}(\xi \rho) d \xi, \quad G(\rho)=\int_{0}^{\infty} \psi(\xi) J_{v}(\xi \rho) d \xi
$$

A standard problem in the theory of dual integral equations is to determine the function $\psi(\xi)$ such that

$$
F(\rho)=f(\rho) \quad(0 \leqq \rho \leqq 1), \quad G(\rho)=g(\rho) \quad(\rho>1),
$$

when the functions $f(\rho), g(\rho)$ are prescribed.

The case in which $g(\rho) \equiv 0$ has been considered by several authors (see references [1], [2], [3]). Recently Copson [4] has given a simple and elegant solution of this pair by a method which is a generalization of an elementary method suggested by Sneddon [5].

The solutions of the pair of equations (2) with $n= \pm 1$ was given by Tranter [6] but his method is rather cumbersome. The pair with general values of $n$ in the range $-2<n<2$ was considered by Noble [7], who reduced the problem to that of solving an integral equation. Noble's analysis involves considerable manipulation and cannot be regarded as elementary. More recently, Williams [8] has derived the solution of the pair (2) by a formal application of Mellin transforms. The manipulation here is formally more simple because much of it can be absorbed in the calculation of the Mellin transforms.

In the present paper we shall consider the equations

$$
F(\rho)=0 \quad(0 \leqq \rho \leqq 1), \quad G(\rho)=g(\rho) \quad(\rho>1),
$$

since once the solution of this pair of equations is known the solution of the pair (2) can be obtained merely by adding Copson's solution of the pair (2) with $g(\rho) \equiv 0$. The method we shall employ is a direct extension of Copson's method [4]. As in Copson's paper we shall treat the two cases $0<n<2$ and $-2<n<0$ separately.

It should also be noted that an elementary solution of the pair of equations (3) in the physically interesting case $n=-1$ was given recently by the authors [9].

In the course of the analysis we shall need

LEMMA A. If $\lambda>\mu>-1$,

$$
\int_{0}^{\infty} J_{\lambda}(a \xi) J_{\mu}(b \xi) \xi^{1+\mu-\lambda} d \xi=\left\{\begin{array}{cc}
0 & (0<a<b), \\
\frac{b^{\mu}\left(a^{2}-b^{2}\right)^{\lambda-\mu-1}}{2^{\lambda-\mu-1} a^{\lambda} \Gamma(\lambda-\mu)} & (0<b<a),
\end{array}\right.
$$

$\dagger$ The work described in this paper was done in the Department of Mathematics, Duke University, North Carolina, and was supported by the U.S.A.F.O.S.R., A.R.D.C. under Contract No. AF $18(600)-1341$. 
which is a well known result in the theory of Bessel functions [10, p. 401], and

Lemma B. If $f(x)$ is continuously differentiable in the interval $[1, \infty)$ and $0<\kappa<1$, then the integral equation

$$
f(x)=\int_{x}^{\infty} \frac{\psi(t) d t}{\left(t^{2}-x^{2}\right)^{x}} \quad(x>1)
$$

has solution

$$
\psi(t)=-\frac{2}{\pi} \sin (\kappa \pi) \frac{d}{d t} \int_{t}^{\infty} \frac{u f(u) d u}{\left(u^{2}-t^{2}\right)^{1-\kappa}},
$$

which can be established by simple methods (cf. $[11$, p. 229]).

2. We shall begin by considering the case $0<n<2$. For convenience we shall write $n=2 \alpha$, so that $0<\alpha<1$. If we take $\psi(\xi)$ to be of the form

$$
\psi(\xi)=\xi^{1-\alpha} \int_{1}^{\infty} \phi(t) J_{v+\alpha}(\xi t) d t,
$$

then, writing the integral in the form

$$
\psi(\xi)=-\xi^{-\alpha} \int_{1}^{\infty} \phi(t) t^{v+\alpha-1} \frac{d}{d t}\left[t^{-v-\alpha+1} J_{v+\alpha-1}(\xi t)\right] d t,
$$

and integrating by parts, we find that, if we assume that

$$
\lim _{t \rightarrow \infty} \phi(t)=0,
$$

then

where

$$
\psi(\xi)=\xi^{-\alpha} \phi(1) J_{v+\alpha-1}(\xi)+\xi^{-\alpha} \int_{1}^{\infty} \Phi(t) J_{v+\alpha-1}(\xi t) d t
$$

$$
\Phi(t)=t^{-v-\alpha+1} \frac{d}{d t}\left[t^{v+\alpha-1} \phi(t)\right]
$$

Now, if $0<\alpha<1, v>-1$, it follows from Lemma $A$ that

$$
\int_{0}^{\infty} \xi^{\alpha} J_{v+\alpha-1}(\xi t) J_{v}(\xi \rho) d \xi=0 \quad(0<\rho<t),
$$

so that, if we substitute from equation (5) into the expression (1) for $F(\rho)$ and interchange the order of the integrations, we find that the form (4) automatically satisfies the first equation of the pair (3).

If we now substitute from equation (4) into the expression (1) for $G(\rho)$, interchange the order of the integrations and use Lemma A, we find that, when $\rho>1$,

$$
G(\rho)=\frac{2^{1-\alpha} \rho^{v}}{\Gamma(\alpha)} \int_{\rho}^{\infty} \frac{t^{-v-\alpha} \phi(t) d t}{\left(t^{2}-\rho^{2}\right)^{1-\alpha}},
$$


so that, using the second of the equations (3), we see that $\phi(t)$ is the solution of the integral equation

$$
2^{\alpha-1} \Gamma(\alpha) \rho^{-v} g(\rho)=\int_{\rho}^{\infty} \frac{t^{-v-\alpha} \phi(t) d t}{\left(t^{2}-\rho^{2}\right)^{1-\alpha}}
$$

The solution of this equation follows from Lemma B. Provided that $\rho^{-v} g(\rho)$ and its first derivative are continuous in $[1, \infty)$,

$$
\phi(t)=-\frac{2^{\alpha} t^{\nu+\alpha}}{\Gamma(1-\alpha)} \frac{d}{d t} \int_{t}^{\infty} \frac{\rho^{-v+1} g(\rho) d \rho}{\left(\rho^{2}-t^{2}\right)^{\alpha}} .
$$

Substituting this value into equation (4), we obtain the solution

$$
\psi(\xi)=-\frac{2^{\alpha} \xi^{1-\alpha}}{\Gamma(1-\alpha)} \int_{1}^{\infty} t^{\nu+\alpha} J_{v+\alpha}(\xi t) d t\left(\frac{d}{d t} \int_{t}^{\infty} \frac{\rho^{-v+1} g(\rho) d \rho}{\left(\rho^{2}-t^{2}\right)^{\alpha}}\right),
$$

which is in agreement with the solutions of Noble and Williams (cf. equation (11) of [8]).

3. We shall now consider the case $-2<n<0$. For convenience, we shall write $n=-2 \beta$, so that $0<\beta<1$. In this case we take the form

$$
\psi(\xi)=\xi^{1+\beta} \int_{1}^{\infty} \phi(t) J_{v-\beta}(\xi t) d t
$$

again with the condition that $\phi(t) \rightarrow 0$ as $t \rightarrow \infty$. Writing this integral in the form

$$
\psi(\xi)=\xi^{\beta} \int_{1}^{\infty} t^{-v+\beta-1} \phi(t) \frac{\partial}{\partial t}\left\{t^{v-\beta+1} J_{v-\beta+1}(\xi t)\right\} d t,
$$

and integrating by parts, we find that

$$
\psi(\xi)=-\phi(1) \xi^{\beta} J_{\nu-\beta+1}(\xi)-\xi^{\beta} \int_{1}^{\infty} \Psi(t) J_{v-\beta+1}(\xi t) d t
$$

where

$$
\Psi(t)=t^{v-\beta+1} \frac{d}{d t}\left\{t^{-v+\beta-1} \phi(t)\right\}
$$

If we substitute the form (9) into the expression for $F(\rho)$, interchange the order of the integrations and make use of Lemma A, we find that $F(\rho)=0$ for $0<\rho<1$. On the other hand if we substitute the expression (10) into the expression for $G(\rho)$, we find that, if $\rho>1$,

$$
G(\rho)=-\frac{2^{\beta} \rho^{\nu}}{\Gamma(1-\beta)} \int_{\rho}^{\infty} \frac{t^{\beta-\nu-1} \Psi(t)}{\left(t^{2}-\rho^{2}\right)^{\beta}} d t
$$

so that $\psi(t)$ satisfies the integral equation

$$
\int_{\rho}^{\infty} \frac{t^{\beta-v-1} \Psi(t) d t}{\left(t^{2}-\rho^{2}\right)^{\beta}}=-2^{-\beta} \rho^{-v} g(\rho) \Gamma(1-\beta) .
$$


It follows from Lemma B that, if $\rho^{-v} g(\rho)$ is continuously differentiable in $[1, \infty)$, then

$$
t^{\alpha-\nu-1} \Psi(t)=\frac{2^{1-\beta}}{\Gamma(\beta)} \frac{d}{d t} \int_{t}^{\infty} \frac{\rho^{1-v} g(\rho) d \rho}{\left(\rho^{2}-t^{2}\right)^{1-\beta}} .
$$

Using equation (11), integrating with respect to $t$ and determining the constant of integration from the condition that $\phi(t) \rightarrow 0$ as $t \rightarrow \infty$, we obtain the solution

$$
\phi(t)=\frac{2^{1-\beta} t^{\nu-\beta+1}}{\Gamma(\beta)} \int_{t}^{\infty} \frac{\rho^{1-v} g(\rho) d \rho}{\left(\rho^{2}-t^{2}\right)^{1-\beta}} .
$$

Substituting this value into equation (9), we obtain the solution

$$
\psi(\xi)=\frac{2^{1-\beta} \xi^{1+\beta}}{\Gamma(\beta)} \int_{1}^{\infty} t^{\nu-\beta+1} J_{v-\beta}(\xi t) d t \int_{t}^{\infty} \frac{\rho^{1-v} g(\rho) d \rho}{\left(\rho^{2}-t^{2}\right)^{1-\beta}} .
$$

Again this is in agreement with the solutions of Noble and Williams. (Cf. equation (10) of . $[8])$.

4. In many problems we are more interested in the form of $F(\rho)$ when $\rho>1$ and of $G(\rho)$ when $0<\rho<1$ than we are in the actual form of the function $\psi(\xi)$. We can derive these easily from the above results.

(a) $0<n<2$ : If we substitute the form (5) for $\psi(\xi)$ into equation (1) and use Lemma $\mathrm{A}$, we find that, if $\rho>1$,

$$
F(\rho)=\frac{2^{\alpha}\left(\rho^{2}-1\right)^{-\alpha} \rho^{-v}}{\Gamma(1-\alpha)} \phi(1)+\frac{2^{\alpha} \rho^{-v}}{\Gamma(1-\alpha)} \int_{1}^{\rho} \frac{t^{\nu+\alpha-1} \Phi(t)}{\left(\rho^{2}-t^{2}\right)^{\alpha}} d t .
$$

Now we can write ( 7$)$ in the form

$$
\phi(t)=-\frac{2^{\alpha} t^{\nu+\alpha+1}}{\Gamma(1-\alpha)} \int_{t}^{\infty} \frac{g_{1}(\rho) d \rho}{\left(\rho^{2}-t^{2}\right)^{\alpha}}, \quad g_{1}(\rho)=\frac{d}{d \rho}\left[\rho^{-v} g(\rho)\right]
$$

so that

$$
\phi(1)=-\frac{2^{\alpha}}{\Gamma(1-\alpha)} \int_{1}^{\infty} \frac{g_{1}(\rho) d \rho}{\left(\rho^{2}-1\right)^{\alpha}}
$$

Hence, when $\rho>1$,

$$
F(\rho)=\frac{2^{\alpha} \rho^{-v}}{\Gamma(1-\alpha)} \int_{1}^{\rho} \frac{t^{v+\alpha-1} \Phi(t) d t}{\left(\rho^{2}-t^{2}\right)^{\alpha}}-\frac{2^{2 \alpha} \rho^{-v}\left(\rho^{2}-1\right)^{-\alpha}}{[\Gamma(1-\alpha)]^{2}} \int_{1}^{\infty} \frac{g_{1}(\rho) d \rho}{\left(\rho^{2}-1\right)^{\alpha}},
$$

where $\Phi(t)$ is defined by equations (6) and (7) and $g_{1}(\rho)$ by the second of equations (14).

Similarly, if we substitute from (4) into the expression for $G(\rho)$ and use Lemma A, we find that, when $0 \leqq \rho \leqq 1$,

$$
G(\rho)=\frac{2^{1-\alpha} \rho^{v}}{\Gamma(\alpha)} \int_{1}^{\infty} \frac{t^{-v-\alpha} \phi(t) d t}{\left(t^{2}-\rho^{2}\right)^{1-\alpha}}
$$


(b) $-2<n<0$ : If we substitute from (9) into the expression for $F(\rho)$ and use Lemma A, we find that

$$
F(\rho)=\frac{2^{1-\beta} \rho^{-\nu}}{\Gamma(\beta)} \int_{1}^{\rho} \frac{t^{\nu-\beta} \phi(t) d t}{\left(\rho^{2}-t^{2}\right)^{1-\beta}} \quad(\rho>1) .
$$

Similarly, if we use the form (10) in the definition of $G(\rho)$, we find that, when $0<\rho<1$,

$$
G(\rho)=-\frac{2^{\beta} \rho^{\nu}\left(1-\rho^{2}\right)^{-\beta}}{\Gamma(1-\beta)} \phi(1)-\frac{2^{\beta} \rho^{\nu}}{\Gamma(1-\beta)} \int_{1}^{\infty} \frac{t^{\beta-\nu-1} \Psi(t) d t}{\left(t^{2}-\rho^{2}\right)^{\beta}}
$$

Substituting the value of $\phi(1)$ from (12), we have

$G(\rho)=-\frac{2 \sin \beta \pi}{\pi} \rho^{v}\left(1-\rho^{2}\right)^{-\beta} \int_{1}^{\infty} \frac{\rho^{1-v} g(\rho) d p}{\left(\rho^{2}-1\right)^{1-\beta}}-\frac{2^{\beta} \rho^{v}}{\Gamma(1-\beta)} \int_{1}^{\infty} \frac{t^{\beta-v-1 \Psi}(t) d t}{\left(t^{2}-\rho^{2}\right)^{\beta}} \quad(0<\rho<1)$,

where $\Psi(t)$ is defined by equations (11) and (12).

\section{REFERENCES}

1. E. C. Titchmarsh, Introduction to the theory of Fourier integrals (Clarendon Press, Oxford, 1937), 334-339.

2. I. W. Busbridge, Dual integral equations, Proc. London Math. Soc. 44 (1938), 115-129.

3. A. N. Gordon, Dual integral equations, J. London Math. Soc. 29 (1954), 360-363.

4. E. T. Copson, On certain dual integral equations, Proc. Glasgow Math. Assoc. 5 (1961), 21-24.

5. I. N. Sneddon, The elementary solution of dual integral equations, Proc. Glasgow Math. Assoc. 4 (1960), 108-110.

6. C. J. Tranter, On some dual integral equations, Quart. J. Math. Oxford Ser. (2) 2 (1951), 60-66.

7. B. Noble, Certain dual integral equations, J. Math. Phys. 37 (1958), 128-136.

8. W. E. Williams, The solution of certain dual integral equations, Proc. Edinburgh Math. Soc. 12 (1961), 213-216.

9. M. Lowengrub and I. N. Sneddon, An axisymmetric boundary value problem of mixed type for a half-space, Proc. Edinburgh Math. Soc. 13 (1962), 39-46. 1944).

10. G. N. Watson, A treatise on the theory of Bessel functions (University Press, Cambridge, 1920).

11. E. T. Whittaker and G. N. Watson, $A$ course of modern analysis (University Press, Cambridge,

\section{THE UNIVERSITY}

GLASGOW 